\title{
Investigación en Progreso: Ingeniería de Software en el Desarrollo de Aplicaciones Sensibles al Contexto
}

\author{
Marisa D. Panizzi, Javier H. Lafont, Lucio O. Bravo, Federico Pierro, Mariano M. Gambino, Leticia L. Madeira \\ Instituto de Investigación en Ingeniería de Software Experimental \\ Universidad de Morón \\ Buenos Aires, Argentina \\ marisapanizzi@speedy.com.ar
}

\begin{abstract}
Resumen- Las aplicaciones han ido evolucionando independientemente de cuál sea su dominio de aplicación, actualmente nos encontramos con aplicaciones que pueden brindar servicios de acuerdo a la ubicación, tiempo y perfil del usuario. Este tipo de aplicaciones que brindan estos servicios se las conoce como Aplicaciones Sensibles al Contexto (CA Context Aware), en este tipo de aplicaciones predominan los dispositivos móviles y el perfil del usuario. La evolución varía en función de la forma de uso de la computadora, es decir que se brinden servicios a pedido del usuario o que se brinden servicios de manera automática.
\end{abstract}

Para acompañar la evolución de este tipo de aplicaciones, la Ingeniería de Software ha focalizado sus esfuerzos en aspectos relacionados a separar la aplicación de todo lo concerniente a la captación, interpretación, control y supervisión de la información contextual.

Es decir que desde el punto de vista del diseño, se separe la conducta sensible al contexto de la conducta propia de la aplicación con el propósito de que ambos tipos de aplicaciones pueden evolucionar en forma independiente además de reducir el tiempo de desarrollo en este tipo de sistemas.

Este proyecto presenta una sub línea, la cual orientará sus aportes, en la consolidación de un framework de gestión contextual de dos o tres variables de contexto como por ejemplo: la ubicación, considerando la construcción de la documentación relevante que lo respalde como así también su fase de experimentación con una aplicación que permitirá a los usuarios de la comunidad universitaria UM hacer uso de los servicios ofrecidos por la misma.

La segunda sub línea, se vincula al Área de la Ingeniería de Requisitos, pretendiendo obtener un modelo para la especificación de requisitos de software para sistemas sensibles al contexto.

Palabras Clave - Ingeniería de Software - Aplicaciones sensibles al contexto - Framework - Sistemas pervasivos Computación Ubicua - Requisitos para aplicaciones sensibles al contexto.

\section{JUSTIFICACIÓN DE LA PROPUESTA}

El presente proyecto se ajusta a las líneas de investigación planteadas por la Unidad Académica, el cual tiene como propósito fortalecer el plan de estudios de las carreras de grado Licenciatura en Sistemas e Ingeniería en Informática considerando su transferencia tanto al grupo de docentes como al alumnado. Otro punto fuerte a mencionar es la incorporación de un grupo de docentes con el objetivo de que inicien su proceso de formación como investigadores. En relación a los alumnos se prevee la incorporación de los mismos para que desarrollen sus trabajos de fin de carrera en el ámbito del proyecto.

El proyecto se radica en el Instituto de Ingeniería de Software Experimental de la Unidad Académica.

\section{ESTADO ACTUAL DEL CONOCIMIENTO SOBRE EL TEMA}

La evolución en el desarrollo de hardware permite imaginarnos un futuro en el cual los usuarios serán asistidos por diversos tipos de computadoras para llevar a cabo sus tareas diarias. Para alcanzar dicho objetivo es necesario una revisión general en el desarrollo de aplicaciones, considerando el nivel de interacción hombre-máquina como así también el nivel de funcionalidad. En este tipo de aplicaciones ya no se puede asumir un contexto fijo, como puede ser el lugar de trabajo o el hogar, sino que las situaciones por las que va pasando el usuario (y por ende su computadora) puede variar drásticamente a lo largo del día. Como respuesta a esta evolución se ha comenzado a trabajar en una ingeniería de software que permita generar aplicaciones, cuyo objetivo es adaptarse al contexto en el que se encuentran, para así poder brindar al usuario servicios en base a su situación.

El diseño de una arquitectura que permita aprovechar la información de contexto, está influenciado por los desarrollos en otras áreas de investigación. Una de las principales influencias está dada por la computación móvil (mobile computing), ya que en la mayoría de los casos tendremos que manejar la comunicación (probablemente intermitente) entre dispositivos, así como la heterogeneidad de hardware. En segundo lugar debemos "aumentar" la experiencia del usuario por medio de la utilización de diversos dispositivos, en forma similar a lo que plantea la computación ubicua (ubiquitous computing). Por último, ya que uno de los aspectos de contexto que resultan más relevantes es la ubicación del usuario, aquellas cosas relacionadas con la computación basada en la locación (location based computing) nos serán de suma utilidad [1].

De la revisión de antecedentes respecto a definir qué es un Sistema Sensible al Contexto, se ha encontrado una clasificación en dos grandes grupos. Aquellas que consideran la computación basada en contexto como el software que simplemente utiliza el contexto, y aquellas que la definen como la computación que no sólo utiliza el contexto sino que además es capaz de adaptarse a él [2].

Según [3] la computación sensible al contexto es un paradigma de computación en el cual las aplicaciones y los servicios pueden tomar ventajas de la información contextual tales como: información del usuario, de la ubicación del dispositivo, del estado del dispositivo, del tiempo, del día, de la interacción usuario-servicio y sus actividades, entre otros.

¿Cuál es la relación entre computación ubicua/ pervasiva y los sistemas sensibles al contexto? La computación sensible al contexto consiste de dispositivos computacionales integrados en el medio ambiente 'rodeando' a las personas de modo tal, que proporcionan servicios aumentados con capacidades sensoriales: los dispositivos situados en un espacio físico 
pueden ver u oír (sentir) quién o qué se encuentra alrededor, qué actividad está realizando, la ubicación física de los objetos o personas del entorno y cuándo está ocurriendo algo [4].

De algún modo los sistemas sensibles al contexto plantean una extensión de la visión presentada por [5]. Son dispositivos dispersos en el entorno del usuario que cooperan con algún software, brindando información del contexto en que se ejecuta la aplicación, permitiendo de este modo al sistema reaccionar en función de los cambios producidos en su entorno [4]

Se han revisado algunos desarrollos realizados en el área, para así poder comprender a que nos enfrentamos, cuáles han sido los problemas encontrados y las soluciones propuestas hasta el momento. Se comenzó revisando algunos ejemplos de aplicaciones context-aware concretas que ilustren cómo se puede utilizar el contexto para brindar servicios al usuario o mejorar la funcionalidad de aplicaciones existentes, entre las que se pueden mencionar: CyberGuide [6], este tipo de aplicaciones que ayudan al usuario durante su recorrido se encuentran probablemente dentro de las aplicaciones contextaware más desarrolladas, ya que han sido objeto de investigación en diversas implementaciones [7, 8, 9, 10, 11]. Otro desarrollo que se consideró es: Forget-me-not [12], el cual se encuentra dentro de un conjunto de aplicaciones contextaware que se utilizan para aumentar la memoria de su usuario.

Otro aplicación que se revisó es: In/Out Board [13], la cual es una aplicación sencilla que se utiliza para saber que personas se encuentran dentro o fuera de un edificio y, dependiendo de esta información, su hora de entrada o salida. Por último podemos mencionar: la aplicación Conference Assistant [14] es probablemente una de las más complejas que se han planteado hasta el momento en el desarrollo de sistemas context-aware. El objetivo de esta aplicación es ayudar a una persona en su asistencia a una conferencia.

Las aplicaciones sensibles al contexto según Gartner aparecerán en algunas áreas determinadas, tales como servicios basados en ubicación, en la realidad aumentada en dispositivos móviles y el comercio electrónico a través del teléfono móvil.[15 ].

Se han encontrado antecedentes relacionados a las propuestas de los frameworks en los cuales se implementan modelos de arquitecturas para las aplicaciones sensibles al contexto. Podemos mencionar las siguientes propuestas: el modelo de Finkesltein que propone las aplicaciones web basadas en reglas (2002), el modelo Winograd que propone un modelo centrado en los datos más que en los procesos (2001), la propuesta de Sivia Gordillo (2006) de una aproximación al diseño para Ingeniería de servicios sensibles al contexto basado en capas y los mecanismos de dependencia en orientación a objetos y la aproximación para diseñar servicios sensibles a la ubicación y describen cómo extender las aplicaciones con un nuevo comportamiento sensible al contexto [1].

\section{OBJETIVO DEL PROYECTO}

El proyecto considera la incorporación de esta línea de investigación en la Unidad Académica con el propósito de que el grupo de investigación madure en Ingeniería de Software en el desarrollo de aplicaciones sensibles al contexto. Este trabajo propone dos objetivos, cada uno de ellos con un enfoque diferente, estos enfoques están claramente definidos en función de quienes harán uso de los resultados del proyecto, es decir profesionales de sistemas y usuarios finales.

El primer objetivo consiste en la consolidación de un framework de gestión contextual de dos o tres variables del contexto. Para el cumplimiento del primer objetivo, se tomará como base la tesis de grado desarrollada por Hernán Guasti y Sebastián de Jesus, actualmente docentes de la Unidad Académica. En su trabajo de tesis se propuso una arquitectura de framework de gestión contextual en la cual se consideró la variable de ubicación/localización, principalmente en ambientes cerrados. Se desarrolló una implementación con alcance limitado (prototipo experimental) donde se puso especial énfasis en la variable de contexto "ubicación" por medio de la gestión de señales WiFi presentes en el radio de alcance del dispositivo móvil. Considerando como punto de partida dicho pseudo framework, se pretende incorporar más variables del contexto, dos o tres variables del contexto a la que ya posee. Dentro de este objetivo se contempla el desarrollo de la documentación de los requisitos, modelos de análisis y diseño correspondientes como así también su fase experimental con una aplicación desarrollada para tal fin. En el marco de este objetivo, en el cual se pretende obtener resultados para los profesionales de sistemas, se incluye el modelo de especificación de requisitos de aplicaciones sensibles al contexto.

El segundo objetivo consiste en desarrollar una aplicación sensible al contexto para la UM, la cual permitirá a los usuarios de la comunidad universitaria UM hacer uso de los servicios ofrecidos por la misma. Dentro de este objetivo se contempla la construcción de la documentación de los requisitos, los modelos de análisis y diseño como así también las pruebas necesarias.

\section{METODOLOGÍA DEL DESARROLLO}

En la actualidad no solo se investigan problemas, oportunidades y necesidades (PON). Un problema se define como una situación conflictiva en la que es posible identificar los elementos que la causan, cuando esto se logra se tienen elementos para un buen planteamiento del PON. El planteamiento del PON es una etapa de suma importancia, de no existir un PON planteado no es posible realizar una investigación científica. De acuerdo con Heinz Dieterich (1997) "el planteamiento del problema es la delimitación clara y precisa del objeto de investigación...." El PON es el motor que impulsa la investigación científica. Un PON bien planteado representa un avance del $50 \%$ en un proceso de investigación (Ackoff, 1933). Se utilizarán las siguientes etapas globales:

- Planteamiento del problema.

- Formulación de hipótesis.

- Comprobación de hipótesis.

- Conclusiones.

La metodología específica a emplear se plantea del siguiente modo:

- Estudio del estado del arte.

- Análisis de la información recabada y un estudio comparativo de los datos obtenidos.

- Formulación de un modelo adecuado al tema a tratar.

- Evaluación de la aplicación de una solución al modelo.

- Construcción de una herramienta de experimentación.

- Construcción de una herramienta definitiva.

Esta división de las tareas parece adecuada al problema, y sigue los lineamientos recomendados para el desarrollo de un proyecto de estas características.

\section{CONTRIBUCION AL AVANCE DEL CONOCIMIENTO CIENTÍFICO Y/O TECNOLÓGICO}

El mayor inconveniente que se presenta en el desarrollo de sistemas sensibles al contexto está relacionado a la gran cantidad de plataformas en las que las aplicaciones móviles pueden ser desplegadas, este tipo de sistemas puede estar 
definido por un conjunto de aplicaciones que se ejecutan en entorno heterogéneo de plataformas tanto de dispositivos como de medios de comunicación.

Este problema que se presenta con el desarrollo de este tipo de aplicaciones es un tema que se viene investigando, que hay antecedentes de su evolución pero que aún queda un espacio en el cual se puede seguir aportando conocimiento.

Con este trabajo se pretende consolidar un framework que permita a los desarrolladores de aplicaciones sensibles al contexto que puedan hacer uso de los datos del contexto a través del uso de dicho framework y de esta manera reducir el tiempo de desarrollo de este tipo de aplicaciones.

El presente trabajo de investigación pretende mostrar que mediante la utilización de un framework que maneje la informacion del contexto de manera independiente a la aplicación se puede reducir el tiempo de desarrollo de sistemas sensibles al contexto.

Dado que la Ingeniería de Requisitos para aplicaciones sensibles al contexto es un tema que ha sido abordado pero que actualmente plantea un área de vacancia a la cual se pueden realizar aportes significativos, se ha considerado la construcción de un modelo para especificar requisitos para este tipo de aplicaciones.

\section{CONDICIONES INSTITUCIONALES PARA EL DESARROLLO DEL PROYECTO DE INVESTIGACIÓN}

Este proyecto se ajusta a las líneas de investigación de la Facultad de Informática, Ciencias de la Comunicación y Técnicas Especiales, se radica en el Instituto de Investigación en Ingeniería de Software Experimental (IESE-UM) de la Universidad de Morón.

Este proyecto cuenta con financiamiento de la Facultad de Informática, Ciencias de la Comunicación y Técnicas Especiales de la Universidad de Morón.

\section{REFERENCIAS}

[1] Fortier, Andrés (2005). "Un enfoque orientado a objetos para software context-aware". Tesis de Licenciatura en Informática.. Universidad Nacional de La Plata. 117 pp.

[2] A. K. Dey, G. D. Abowd, Towards a better understanding of context and context-awareness, Workshop on the What, Who, Where, When, and How of Context-Awareness, The Hague, the Netherlands, Abril 2000.

[3] A. Galis, A. Juhola, D. Raz, J. Serrat-Fernandez, Fast and Efficient Context-Aware Services, John Wiley \& Sons Ltd2006.

[4] Castelli, Viviana, Thomas, Pablo y Bertone Rodolfo. " Ingeniería de Requerimientos para Sistemas Sensibles al Contexto, un estudio comparativo". XIV Congreso Argentino de Ciencias de la Computación. (CACIC). Chilecito. 6 al 10 de octubre 2008- Argentina. ISBN 978-987-24611-0-2.

[5] http://www.ubiq.com/hypertext/weiser/UbiCompHotTopics.htm 1

[6] Sue Long, Rob Kooper, Gregory D. Abowd, and Christopher G. Atkeson. Rapid prototyping of mobile context-aware applications: The cyberguide case study. In MOBICOM, pages 97-107, 1996.

[7] Benjamin B. Bederson. Audio augmented reality: a prototype automated tour guide. In CHI 95 Conference Companion, pages 210-211, 1995.

[8] N. Davies, K. Mitchell, K. Cheverst, and G. Blair. Developing a context sensitive tourist guide, 1998.

[9] Steven Feiner, Blair MacIntyre, Tobias Hollerer, and Anthony Webster. A touring machine: Prototyping $3 \mathrm{~d}$ mobile augmented reality systems for exploring the urban environment. In ISWC '97: Proceedings of the 1st IEEE International Symposium on
Wearable Computers, page 74, Washington, DC, USA, 1997. IEEE Computer Society.

[10] Yasuyuki Sumi, Tameyuki Etani, Sidney Fels, Nicolas Simonet, Kaoru Kobayashi, and Kenji Mase. C-map: Building a contextaware mobile assistant for exhibition tours. In Community Computing and Support Systems, pages 137-154, 1998.

[11] Jie Yang, Weiyi Yang, Matthias Denecke, and Alex Waibel. Smart sight: A tourist assistant system. In ISWC '99: Proceedings of the 3rd IEEE International Symposium on Wearable Computers, page 73, Washington, DC, USA, 1999. IEEE Computer Society.

[12] M. Lamming and M. Flynn. Forget-me-not: intimate computing in support of human memory. In Proceedings FRIEND21 Symposium on Next Generation Human Interfaces, 1994.

[13] Daniel Salber, Anind K. Dey, and Gregory D. Abowd. The context toolkit: Aiding the development of context-enabled applications. In CHI, pages 434-441, 1999.

[14] Anind K. Dey, Daniel Salber, Gregory D. Abowd, and Masayasu Futakawa. The conference assistant: Combining context-awareness with wearable computing. In ISWC, pages 21-28, 1999.

[15] http://soliumblog.controlf5.es/tecnologias-estrategicasaplicaciones-moviles-y-experiencia-del-usuario-mas-social/

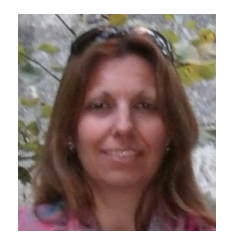

Marisa Daniela Panizzi. Licenciada en Sistemas (Orientación Sistemas Informáticos) de la Universidad de Morón (1998). Profesora Universitaria en Sistemas de Información, Universidad Del Salvador (2006) y Magister en Informática de la Universidad Nacional de La Matanza (2012). Profesora del Seminario de Modelado Conceptual de la Maestría en Ingeniería en Sistemas de Información, Escuela de Postgrado de la UTN-FRBA. Docente de las asignaturas de tesis en la Universidad de Morón y Docente de grado en la UTN FRBA. Directora del Instituto de Investigación en Ingeniería de Software Experimental (Universidad de Morón). Directora del PID: "Ingeniería de software en el desarrollo de aplicaciones sensibles contexto“. Universidad de Morón. (Período 2014-2016).

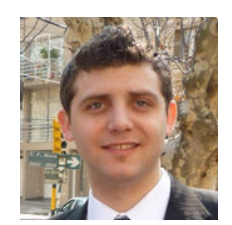

Javier Hernán Lafont. Licenciado en Tecnologías de la Información de la Universidad de Morón (2012). Ayudante de lsa asignaturas de tesis de grado en la Universidad de Morón. Especialista en Ensayos No Destructivos de la Universidad Nacional de San Martín (2013). Integrante del Instituto de Investigación en Ingeniería de Software Experimental (Universidad de Morón). Investigador en el Proyecto "Ingenieria de Software en el desarrollo de aplicaciones sensibles al contexto“. Universidad de Morón. (Período 2014-2016)

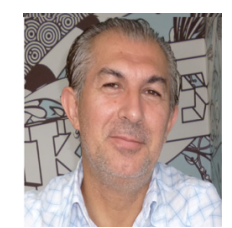

Lucio Oscar Bravo. Licenciado en Sistemas (Orientación Sistemas Informáticos) de la Universidad de Morón (1996). Profesor Universitario en Sistemas de Información, Universidad Del Salvador (2006). Tesis en curso de la Maestria en Informática de la Universidad Nacional de La Matanza. Docente de grado en la Universidad de Morón, UTN FRBA y UAI. Integrante del Instituto de Investigación en Ingeniería de Software Experimental (Universidad de Morón). Investigador en el Proyecto: "Ingeniería de software en el desarrollo de aplicaciones sensibles contexto". Universidad de Morón. (Período 2014-2016). En el marco del proyecto se encuentra desarrollando su tesis de Maestria. 


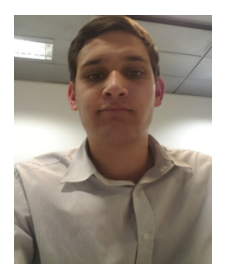

Federico Pierro. Analista en Informática de la Universidad de Morón (2012). Ingeniero en Informática de la Universidad de Morón (2015). Estudiante avanzado de la carrera Ingenieria en Informática. Integrante del Instituto de Investigación en Ingeniería de Software Experimental (Universidad de Morón), en el cual es el responsable del Proyecto de Repositorio Digital para los trabajos de tesis de la carreras del Área de Informática. Investigador en el Proyecto: "Ingeniería de software en el desarrollo de aplicaciones sensibles contexto". Universidad de Morón. (Período 2014-2016).

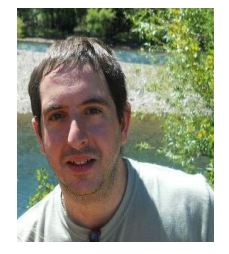

Mariano Martin Gambino. Estudiante avanzado de la carrera Ingeniería en Informática. Integrante del Instituto de Investigación en Ingeniería de Software Experimental (Universidad de Morón). Colaborador en el Proyecto: "Ingeniería de software en el desarrollo de aplicaciones sensibles contexto“. Universidad de Morón. (Período 20142016). En el ámbito del proyecto se encuentra desarrollando su tesis de grado de la carrera Ingenieria en Informática.

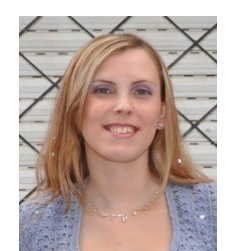

Leticia Lorena Madeira. Licenciatura en Sistemas de la Universidad de Morón (2015). Integrante del Instituto de Investigación en Ingeniería de Software Experimental (Universidad de Morón). Colaboradora en el Proyecto: "Ingeniería de software en el desarrollo de aplicaciones sensibles contexto". Universidad de Morón. (Período 2014-2016). 